\title{
Tratamiento endourológico de la estenosis pieloureteral congénita
}

\author{
Parente Hernández A, Angulo Madero JM, Romero Ruiz R, Cañizo López A, Laín Fernández A, \\ Vázquez Estévez J.
}

Servicio Cirugía Pediátrica. Hospital Infantil Gregorio Marañón. Madrid.

Actas Urol Esp. 2006;30(9):933-938

\section{RESUMEN}

TRATAMIENTO ENDOUROLÓGICO DE LA ESTENOSIS PIELOURETERAL CONGÉNITA

Objetivo: Presentamos nuestra experiencia en el tratamiento endourológico de la estenosis pieloureteral (EPU) en niños.

Material y Métodos: Revisamos 7 pacientes con EPU congénita (edades comprendidas entre 9 meses y 15 años) tratados mediante dilatación endourológica en nuestro centro desde Julio de 2004 . El tratamiento se realizó mediante dilatación endourológica retrógrada bajo control radioscópico. En todos se realizó cistoscopia, se tutorizó el uréter $(4 \mathrm{Fr}$ ó $5 \mathrm{Fr}$ ), realizándose pielografia retrógrada y colocación de guía ureteral de 0,014 “ ó 0,035“. Se realizó la dilatación de la EPU mediante balón de alta presión con perfil de 3Fr ó $5 \mathrm{Fr}$. Se colocaron stents tipo doble J de 3Fr, 4Fr, 4,8Fr ó $6 \mathrm{Fr}$, manteniéndolo durante 6 semanas.

Resultados: En ningún caso hubo complicaciones intraoperatorias. La mediana de estancia hospitalaria fue 2 días (2-8 días). La retirada del stent tipo doble $J$ se llevó a cabo sin incidencias a las 6 semanas de forma ambulatoria. Todos los pacientes permanecen asintomáticos, con disminución del diámetro anteroposterior de pelvis en el seguimiento ecográfico y mejoría del patrón obstructivo en el renograma.

Conclusiones: El tratamiento de la EPU congénita mediante dilatación endourológica retrógrada es posible incluso por debajo del año de edad. La estancia hospitalaria es mínima, sin existir complicaciones intraoperatorias.

Palabras clave: Estenosis pieloureteral. Dilatación balón. Cirugía endourológica.

\section{ABSTRACT}

MANAGEMENT ENDOUROLOGIC OF PYELOURETERAL JUNCTION STENOSIS

Objetives: To report our experience with endourologic methods in the treatment of pyeloureteral stenosis in children.

Materials and Methods: From July 2004, 7 patients from 9 months to 15 years old with pyeloureteral junction stenosis (PUJ) diagnosis underwent endourologic repair. An endourological dilatation was made under radioscopic control. The procedures consist of an initial cystoscopy with a retrograde placement of catheter $\left(4\right.$ or $5 \mathrm{Fr}$ ) and the guide wire $\left(0,014^{\prime \prime}\right.$ or $\left.0,035^{\prime \prime}\right)$. The high pressure balloon (3 to 5 Fr) was then railroad over the wire and positioned across the stenosis for dilatation. A double $\mathrm{J}$ stent ( 3 to $6 \mathrm{Fr}$ ) was then introduced, remaining it during 6 weeks.

Results: There was no intraoperative complications. Median inhospital stay was 2 days (range, 2 to 8). The double $J$ stent was removed without complications. All patients are asymptomatic with improvement in excretion times (MAG3 renography) and antero-posterior renal pelvis diameter (ultrasound measurement).

Conclusions: Balloon dilatation is a viable option in the management of PUJ obstruction even in children under one year old, with minimal morbidity.

Keywords: Pyeloureteral stenosis. Balloon dilatation. Endoluminal surgery. 
$\mathrm{L}$ a unión pieloureteral es la localización más frecuente de obstrucción en el tracto urinario $^{1}$. La obstrucción puede ser detectada en cualquier momento de la vida, aunque el avance en las técnicas de diagnóstico prenatal ha incrementado el número de casos diagnosticados en los primeros momentos de la vida. Además, un importante número de niños son diagnosticados en la infancia debido a que la obstrucción de la unión pieloureteral puede causar clínica desde su inicio, habitualmente en forma de dolor abdominal recurrente, infecciones urinarias de repetición o hematuria. Aunque la causa de la obstrucción puede ser extrínseca (vasos aberrantes como causa más frecuente), en su mayoría están originados por una obstrucción congénita intrínseca $^{2,3}$

Cuando el carácter obstructivo de la estenosis pielouereteral congénita está totalmente demostrado, el tratamiento debe ser quirúrgico ${ }^{4-7}$. Hasta el momento la pieloplastia desmembrada es el "gold estándar" de los tratamientos quirúrgicos en niños. En la actualidad existen técnicas endourológicas que permiten tratar la estenosis pieloureteral $^{8}$. La pieloplastia laparoscópica está aumentando su aceptación entre los cirujanos pediátricos a la vez que aumenta su porcentaje de éxito ${ }^{9}$. La dilatación endourológica con balón, técnica menos invasiva que las anteriores y más utilizada en adultos, puede tener su aplicación en el tratamiento de la estenosis pieloureteral congénita en niños ${ }^{10-15}$. La dilatación con balón de la estenosis fue descrita por primera vez en 1982 por Kandir et al. ${ }^{16}$. Puede ser realizada retrógradamente, mediante cistoscopia, o bien anterógradamente, de forma percutánea o a través de una nefrostomía. Presentamos nuestra experiencia en el tratamiento endourológico de la EPU en niños mediante dilatación retrógrada con balón, con especial atención a los pacientes lactantes.

\section{MATERIAL Y MÉTODOS}

Se han estudiado retrospectivamente los pacientes con estenosis pieloureteral tratados mediante dilatación endourológica en nuestro centro desde Julio de 2004. Se han analizado un total de 7 pacientes, 5 niños y 2 niñas, con EPU congénita, con edades en el momento del tratamiento entre 8 meses y 15 años (media 6,1 años). Tres de los pacientes tenían diagnóstico prenatal de hidronefrosis y fueron tratados antes del año de edad. En el resto de pacientes el estudio se inició por dolor abdominal recurrente ( 2 pacientes), hematuria (1 paciente) e infecciones urinarias de repetición (1 paciente). El diagnóstico se realizó mediante ecografía abdominal (con medición del diámetro anteroposterior de pelvis), urografía intravenosa y renograma diurético MAG-3 (mercapto-acetil-triglicina) III en todos los casos. El tratamiento se realizó mediante dilatación endourológica retrógrada bajo control radioscópico. En todos los casos se realizó cistoscopia (Storz 10) localizándose ambos meatos ureterales. Se tutorizó el uréter mediante sondas ureterales de 4 Fr o 5 Fr (Tutor ureteral punta abierta, Optimed), realizándose pielografía retrógrada y objetivándose la localización de la estenosis pieloureteral. Se colocaron guías ureterales hasta pelvis renal a través del catéter ureteral de 0,014“- (PT2, Boston Scientific) en los pacientes menores de 2 años (3 casos) y de 0,035“ (Uriope, Porges) en los pacientes mayores de 2 años (4 casos). Se procedió a la dilatación de la estenosis pieloureteral mediante balones de alta presión con perfil de 3 Fr (Maverick $^{2}$, Boston Scientific) en los 3 pacientes menores de 1 año ó 5 Fr (Quadrimatrix, Optimed; Uromax Ultra, Boston Scientific) en los 4 pacientes restantes sobre la guía ureteral. Los diámetros de inflado alcanzados variaron desde $4,8 \mathrm{~mm}$ hasta $8 \mathrm{~mm}$, siendo de 4,8 $\mathrm{mm}(\mathrm{n}=1)$ y $5 \mathrm{~mm}(\mathrm{n}=2)$ en los 3 pacientes lactantes. La dilatación fue controlada en todos los casos por radioscopia y mediante control de las presiones de inflado. Se consideró dilatado al desaparecer la muesca del balón en la radioscopia (Fig. 1). En 2 pacientes se produjo extravasación extraluminal del contraste. Tras la dilatación se colocaron stents tipo doble $\mathrm{J}$ en 6 casos (Fig. 2). El diámetro y longitud utilizados fue elegido en función de la edad del paciente, siendo de 3 Fr ó 4 Fr (Optisoft, Optimed) en los pacientes menores de 1 año. En el resto se utilizaron stents de 4,8 Fr (Vortek, Porges) en un paciente de 8 años ó 6 Fr (Stent ureteral silicona, Porges) en los mayores de 10 años. En 1 paciente se recolocó su nefrostomía previa. Se recogieron las complicaciones intraoperatorias, postoperatorias precoces y postoperatorias tardías, así como los requerimientos analgésicos, el tiempo 

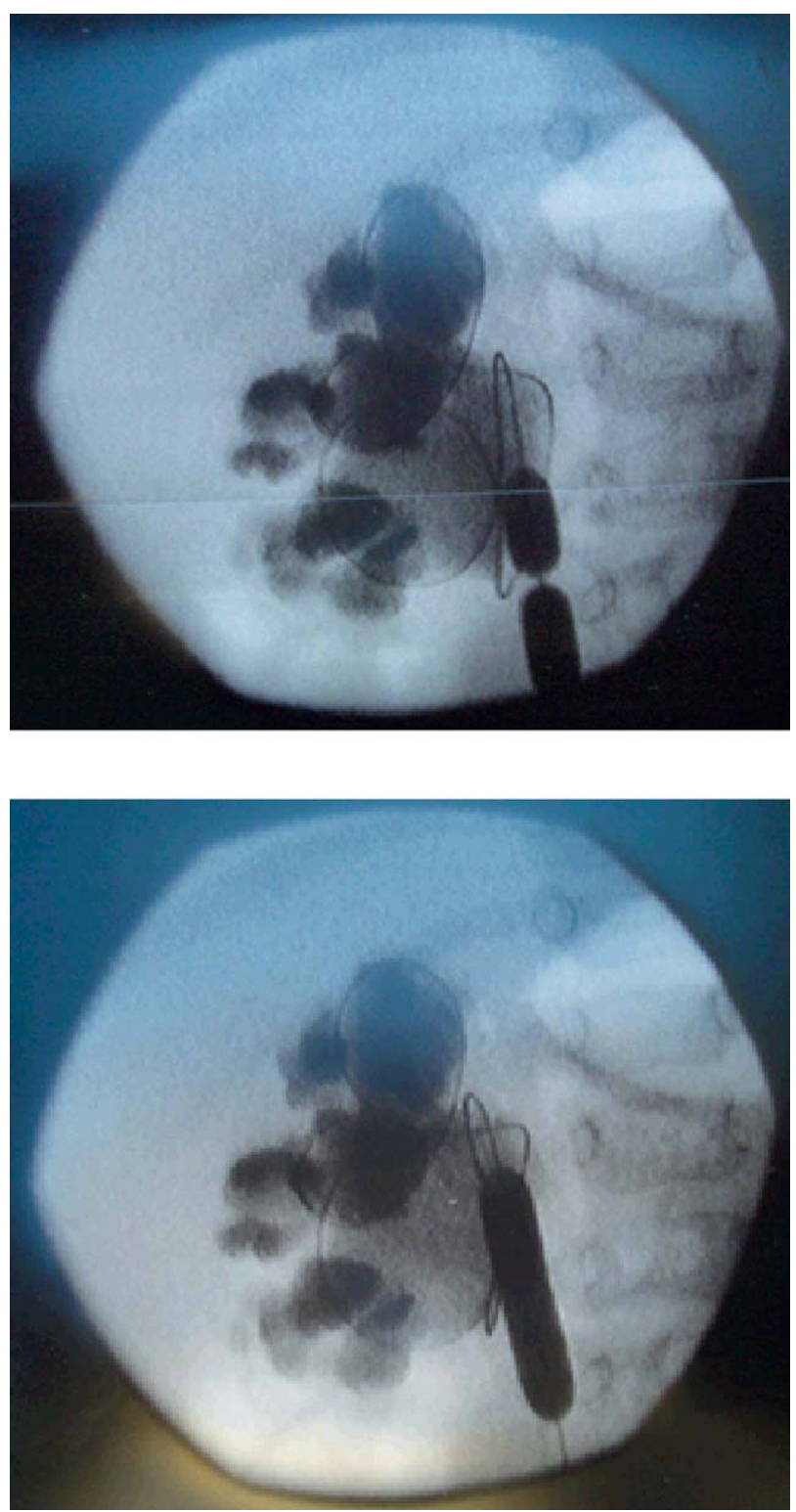

FIGURA 1: Dilatación con balón de estenosis pieloureteral en paciente 8 meses.

quirúrgico y el tiempo de estancia hospitalaria. El seguimiento postoperatorio se realizó mediante ecografia abdominal previa a la retirada del stent, que se repitió a los 2 meses de la retirada. Además se realizó renograma diurético $\mathrm{MAG}-3$ al $6^{\circ}$ mes postoperatorio.

\section{RESULTADOS}

En todos los casos existía una dilatación del diámetro anteroposterior de la pelvis renal en la ecografia e hidronefrosis grado III-IV y una estenosis de la unión pieloureteral comprobada median- te urografía intravenosa. La indicación del tratamiento quirúrgico se realizó por un patrón obstructivo de la curva en el renograma con un tiempo medio de eliminación (T1/2) mayor de 20 en 6 de los casos. En un caso, varón de 11 años, la indicación se realizó por dolor abdominal recurrente. Las ecografias renales en los periodos de dolor presentaban un aumento de 1,5 veces el diámetro anteroposterior de la pelvis renal en comparación con los periodos intercríticos. En este paciente el patrón de la curva en el renograma diurético era semiobstructivo y su T1/2 de 13.
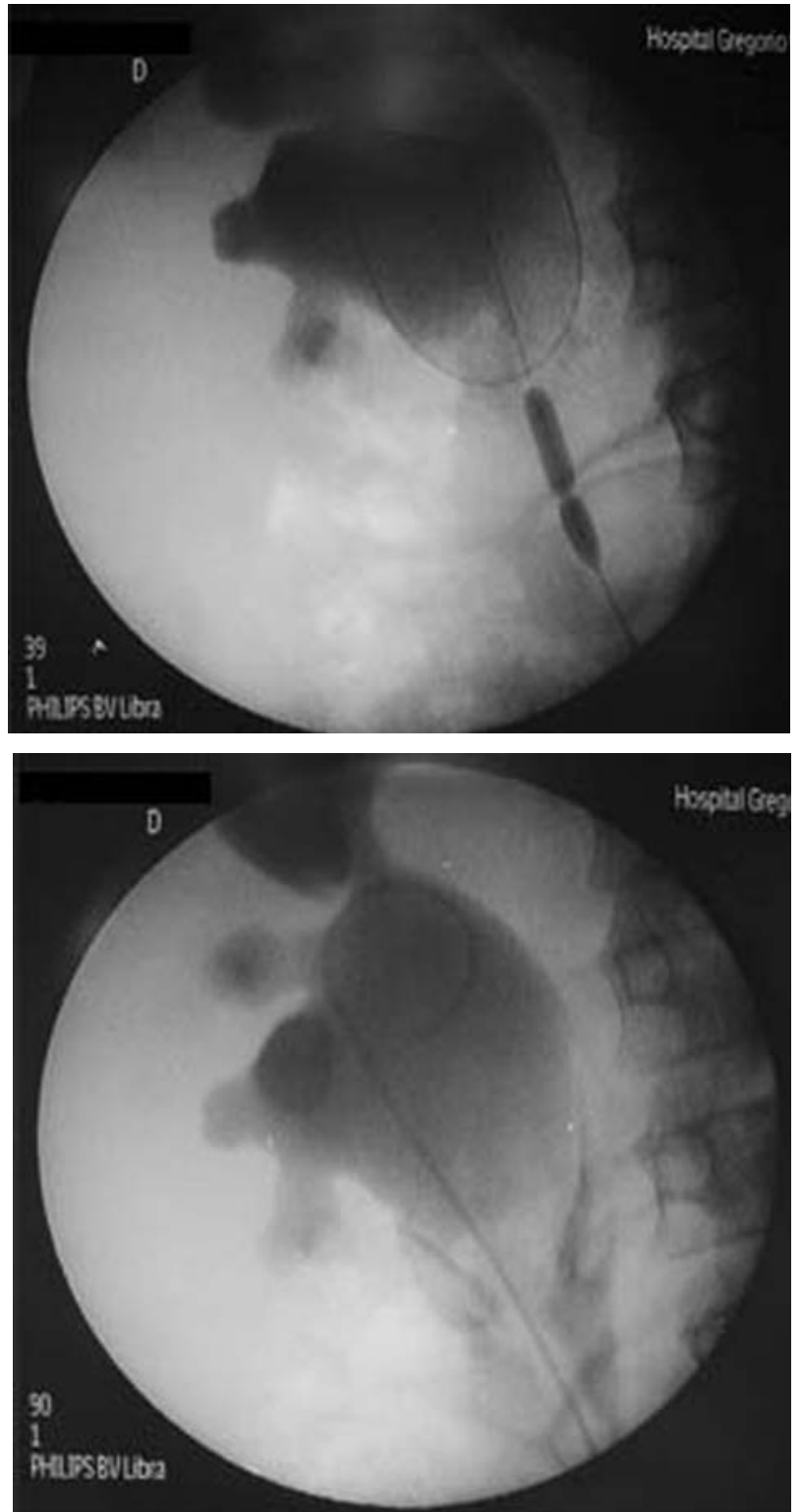

FIGURA 2: Dilatación con balón de EPU observándose extravasación de contraste y colocación de doble $J$. 
En ningún caso hubo complicaciones intraoperatorias. La media de tiempo operatorio fue 51,6 minutos (rango, 43' a 71'). El tiempo de estancia hospitalaria fue de 2 días en 5 de los pacientes. En un paciente, varón de 11 años, fue de 8 días por mal control del dolor postoperatorio. En otro paciente, niña de 15 años, fue de 6 días por obstrucción del stent ureteral que requirió de nefrostomía temporal. Los 5 pacientes menores de 9 años requirieron exclusivamente AINES en el postoperatorio inmediato para control del dolor. Los 2 pacientes mayores de 9 años necesitaron analgesia vía epidural con bupivacaína a bajas dosis administrada mediante perfusión continua más bolos asociada a AINES. En ningún caso aparecieron complicaciones en el periodo posterior al alta hospitalaria. La retirada del stent tipo doble $\mathrm{J}$ se llevó a cabo sin incidencias en todos los casos a las 6 semanas del procedimiento. Esta se realizó de forma ambulatoria, sin necesidad de ingreso hospitalario. Durante la retirada se realizó pielografía retrograda y calibración de la unión pieloureteral, sin necesidad de realizar nueva dilatación en ningún caso. Se realizó cultivo de la punta del stent tipo doble $\mathrm{J}$ siendo estéril en 5 casos y positivo en 2 casos (Streptococo Viridans en el primero y Enterobacter Cloacae en el segundo). Todos los pacientes permanecen asintomáticos, con disminución del diámetro anteroposterior de pelvis en el seguimiento ecográfico en 6 de los casos y mejoría del patrón obstructivo en el renograma en todos ellos. El periodo medio de seguimiento fue de 18,42 meses (rango, 12 a 24) (Tabla 1).

\section{DISCUSIÓN}

El tratamiento de la estenosis pieloureteral mediante dilatación endourológica retrógrada fue inicialmente descrito en adultos. Sin embargo, diversos autores ya lo han desarrollado con éxito en niños. Doraiswamy, utilizando una técnica diferente de la actual (con balón de Fogarty), logra realizar la dilatación en 10 de 11 pacientes, aunque la mayoría requirieron múltiples dilataciones ${ }^{11}$. En nuestra opinión, son necesarias presiones de inflado muy superiores a las alcanzadas con el balón de Fogarty para lograr la dilatación de la obstrucción. Sugita and cols. comienzan a utilizar stent tipo pigtail en muchos de los casos de su serie, obteniendo resultados favorables en el $47 \%$ de los casos $^{12}$. Bolton utiliza el balón Acucise en un intento de mejorar los resultados ${ }^{17}$. Los porcentajes de éxito en las siguientes publicaciones fueron mejorando según se lograba mejorar la técnica, siendo cercanos al $90 \%$ en estudios más recientes ${ }^{14}$. Sin embargo, ninguna de los estudios previos tienen un porcentaje de pacientes por debajo del año de edad valorable, encontrando algunos que incluso dudan de la viabilidad de la técnica en lactantes. En nuestro estudio hemos encontrado que no existen mayor número de complicaciones en paciente menores de 1 año, siendo las necesidades analgésicas y el tiempo de estancia operatoria menores. Las dificultades técnicas deben ser resueltas con un uso de un material quirúrgico adecuado, con perfiles menores de 4 Fr si es necesario, sin necesidad de variar la técnica operatoria. No encontramos diferencias en los resultados en función de la edad del paciente ni del grado de dilatación de la pelvis renal previa a la cirugía, como afirman algunos autores ${ }^{18}$.

Tabla 1

Datos ecográficos, renográficos y clínicos preoperatorios y postoperatorios de los pacientes tratados

\begin{tabular}{|c|c|c|c|c|c|c|c|c|c|c|c|}
\hline Paciente & $\begin{array}{c}\text { DAP } \\
\text { pelvis } \\
\text { pre- } \\
\text { operatorio }\end{array}$ & $\begin{array}{c}\text { DAP } \\
\text { pelvis } \\
\text { post } \\
\text { operatorio }\end{array}$ & $\begin{array}{c}\text { T1/2 } \\
\text { pre- } \\
\text { operatorio }\end{array}$ & $\begin{array}{c}\mathrm{T} 1 / 2 \\
\text { post } \\
\text { operatorio }\end{array}$ & $\begin{array}{c}\text { Tmáx } \\
\text { pre- } \\
\text { operatorio }\end{array}$ & $\begin{array}{c}\text { Tmáx } \\
\text { post- } \\
\text { operatorio }\end{array}$ & $\begin{array}{c}\text { Patrón } \\
\text { curva } \\
\text { pre- } \\
\text { operatorio }\end{array}$ & $\begin{array}{c}\text { Patrón } \\
\text { curva } \\
\text { post- } \\
\text { operatorio }\end{array}$ & $\begin{array}{c}\text { Clinica } \\
\text { pre- } \\
\text { operatoria }\end{array}$ & Seguimiento & $\begin{array}{c}\text { Clinica } \\
\text { post- } \\
\text { operatoria }\end{array}$ \\
\hline 1 & $16 \mathrm{~mm}$ & $9 \mathrm{~mm}$ & $20^{\circ}$ & $9^{-}$ & $10^{\circ} 40^{\circ}$ & $240 "$ & Obstructivo & Normal & ITUs & 12 meses & Asintomático \\
\hline 2 & $48 \mathrm{~mm}$ & $25 \mathrm{~mm}$ & $20^{\circ}$ & $7399^{\prime \prime}$ & $19^{\circ}$ & $5^{\circ} 20^{\circ}$ & Obstructivo & Normal & Dolor abdominal & 24 meses & Asintomático \\
\hline 3 & $8 \mathrm{~mm}$ & $17 \mathrm{~mm}$ & $20^{\circ}$ & $8^{\circ} 20^{\prime \prime}$ & 840 & $20^{\prime \prime}$ & Semiobstructivo & Normal & Hematuria & 15 meses & Asintomático \\
\hline 4 & $23 \mathrm{~mm}$ & $15 \mathrm{~mm}$ & $20^{\circ}$ & $20^{\circ}$ & $20^{\circ}$ & $15^{\circ}$ & Obstructivo & Semiobstructivo & Dolor abdominal & 13 meses & Asintomático \\
\hline 5 & $18 \mathrm{~mm}$ & $16 \mathrm{~mm}$ & $20^{\circ}$ & $20^{\circ}$ & $20^{\circ}$ & $16^{\circ}$ & Obstructivo & Semiobstructivo & Dolor abdominal & 20 meses & Asintomático \\
\hline 6 & $28 \mathrm{~mm}$ & $11 \mathrm{~mm}$ & $20^{\circ}$ & $7222^{\prime \prime}$ & $18^{\circ}$ & $320 "$ & Obstructivo & Normal & Asintomático & 14 meses & Asintomático \\
\hline 7 & $18 \mathrm{~mm}$ & $11 \mathrm{~mm}$ & $20^{\circ}$ & $11^{\circ}$ & 720 " & 5 & Obstructivo & Normal & ITUs & 17 meses & Asintomático \\
\hline
\end{tabular}


En nuestro protocolo no se incluye de forma rutinaria el estudio de vasos polares, aunque en 2 casos sí fueron realizados: varón de 9 años para comprobar la ausencia de algún vaso aberrante como causa de EPU con hidronefrosis intermitente y niña de 15 años por debut tardío. Existen estudios en los que se muestra que el porcentaje de vasos polares en niños es muy inferior a la edad adulta, disminuyendo éste conforme disminuye la $\operatorname{edad}^{2-3,19}$. Aunque algunos autores realizan ecodoppler o TAC previo a la intervención quirúrgica, Nakada et al. demuestran en un estudio de screening de vaso polar realizado mediante TAC, que el porcentaje de pacientes en los que se encuentran posibles vasos aberrantes es mucho mayor que el porcentaje de pacientes en los que esos vasos causan obstrucción de la unión pieloureteral ${ }^{20}$. Sin embargo, tras el tratamiento endourológico, estos estudios son planteados en los casos de fracaso, especialmente en aquellos en los que no se apreció muesca en el balón durante la dilatación.

En todos los pacientes se utilizó un stent tipo doble $\mathrm{J}$, siendo este retirado de forma protocolizada a las 6 semanas del tratamiento quirúrgico. En nuestra experiencia, es importante realizar una revisión a las 6 semanas de la dilatación, pues es en este momento en el que la retracción que produce la cicatrización es máxima. Aunque no hay estudios amplios sobre el uso del stent en niños, sî se ha demostrado en adultos que, en ausencia de algún tipo de stent, existe un aumento del número de fracasos terapéuticos por obstrucción aguda en el postoperatorio. En nuestra serie, no existe ninguna complicación derivada del uso del stent tipo doble $\mathrm{J}$, siendo realizada su retirada de forma ambulatoria, minimizando las molestias del paciente y su familia. En todos los casos en los que se intentó, fue posible su colocación sin aumentar el tiempo quirúrgico, incluso por debajo del año de edad, utilizando en todos los casos material adecuado a la edad y tamaño del paciente. Es destacable el elevado número de pacientes asintomáticos con cultivos positivos del stent tras su retirada, por lo cual optamos por una retirada precoz.

Aunque el tiempo de seguimiento es corto, los resultados son favorables, con una normalización en el patrón del renograma (Fig. 3), así como en el seguimiento ecográfico en 5 de los casos. En los 2 casos en los cuales existía una mejoría en el
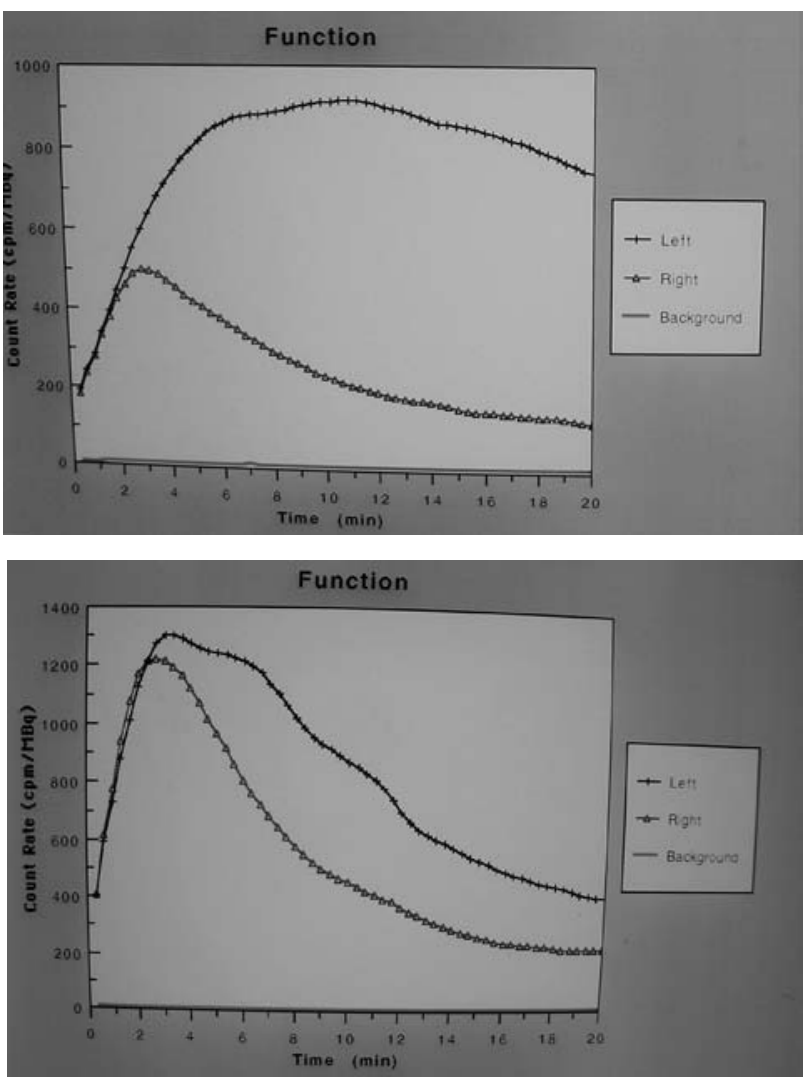

FIGURA 3: Renograma preoperatorio y postoperatorio en paciente con EPU.

patrón renográfico sin normalización completa, se comprobó una clara mejoría ecográfica (Fig. 4) así como en la urografía intravenosa. Todos los pacientes permanecen asintomáticos en el seguimiento (Tabla 1). No hubo complicaciones infecciosas ni reingresos.

Existen publicaciones en las que se comunican casos de reestenosis después de 17 meses de la dilatación ${ }^{14}$, por lo que estos resultados son provisionales y debe ampliarse el seguimiento para obtener resultados más valorables.

El tratamiento de la EPU congénita en niños mediante dilatación es posible incluso por debajo del año de edad. En nuestra experiencia, la dilatación endourológica retrógrada presenta un menor tiempo operatorio, una menor necesidad de analgesia, una menor estancia hospitalaria y un menor porcentaje de complicaciones postoperatorias que la pieloplastia desmembrada, por lo que es una opción que debe ser valorada al plantear el tratamiento quirúrgico de la EPU congénita. Además, como Mackenzie et al. publican en 

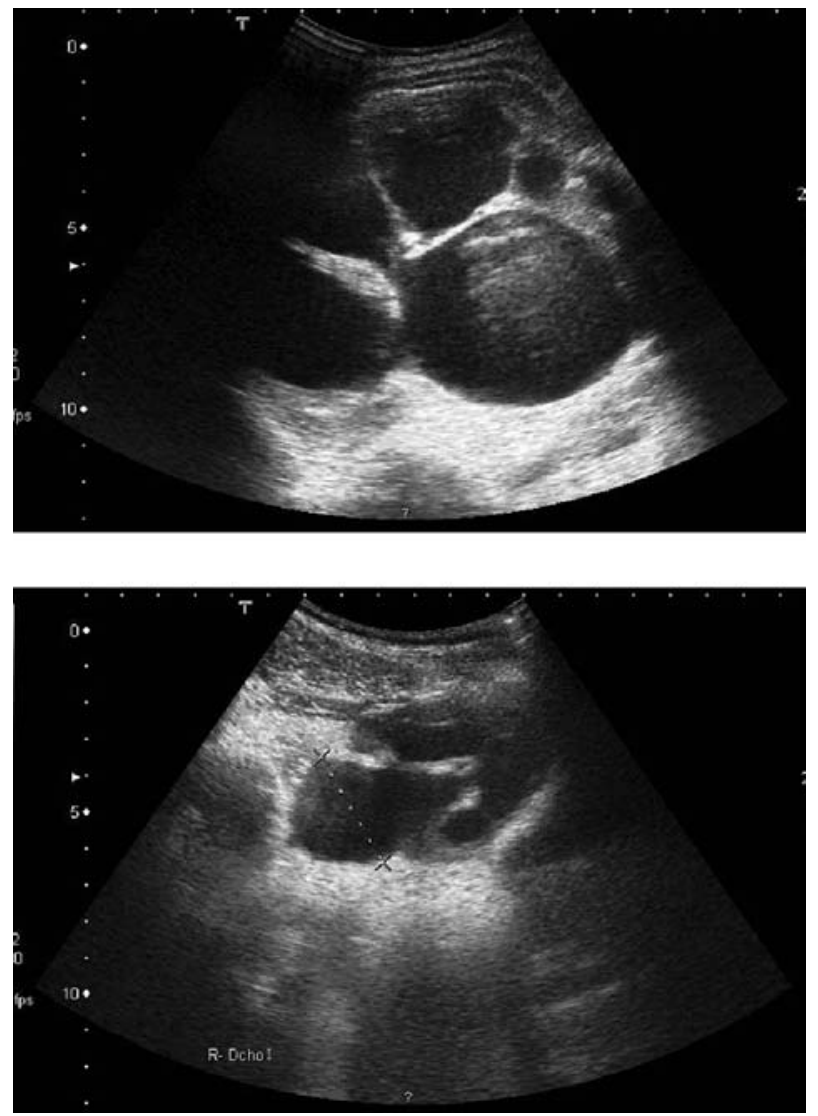

FIGURA 4: Ecografia properatoria y postoperatoria en paciente con EPU.

su serie, el fracaso en la técnica endourológica no compromete la pieloplastia abierta al no modificar el campo quirúrgico, por lo que creemos que no debe ser una segunda opción en la EPU primaria $^{14}$. Se trata de una técnica mínimamente invasiva, cuyos porcentajes de éxito se aproximan a los de la pieloplastia abierta según mejora la experiencia de los autores y se optimiza el material utilizado, por lo que creemos es una técnica terapéutica que debe seguir analizándose como técnica de elección en la EPU congénita.

\section{REFERENCIAS}

1. Perez LM, Friedman RM, King LR: The case for relief of ureteropelvic junction oobstruction in neonates and young chidren at time of diagnosis. Urology 1991;38(3):195-201.

2. Siegel C, McDougall E, Middleton WD, Brink JA, Quillin SP, Teeey SA, et al: Preoperative assessment of ureteropelvic junction obstruction with endoluminal sonography and helical CT. AJR Am J Roentgenol 1997;168(3);623-626.

3. Quillin S, Brink J, Heiken JP, Siegel CL, McClennan BL, Clayman RV: Helical (spiral) CT angiography for identification of crossing vessels at the ureteropelvic junction. AJR Am J Roentgenol 1996; 166(5):1125-1130.
4. Wolpert JJ, Woodart JR, Parrott TS: Pyeloplasty in the young infant. J Urol 1989;144(2 Pt 2):573-575.

5. Gordon I, Dhillon HK, Gatanash H, Peters AM: Antenatal diagnosis of pelvic hydronephrosis: Assessment of renal function and drainage as a guide to management. J Nucl Med 1991;32(9):1649-1654.

6. Koff SA, Campbell KD: The nonoperative management of unilateral neonatal hydronephrosis: Natural histoty of poorly functioning kidneys. J Urol 1994;152(2 Pt 2);593595.

7. Woo HH, Farnsworth HH: Dismembered pyeloplasty in infants under the age of 12 months. Br J Urol 1996;77(3): 449-451.

8. Schenkman E, Tarry W: Comparison of percutaneous endopyelotomy with open pyeloplasty for pediatric UPJ obstruction. J Urol 1998;159(3):1013-1015.

9. Tan HI: Laparoscopic Anderson-hynes dismembered pyeloplasty in children. J Urol 1999;162(2):1045-1048.

10. Tan HL, Roberts JP, Grattan-Smith D: Retrograde balloon dilatation of ureteropelvic obstructions in infants and children: Early results. Urology 1995;46(1):89-91.

11. Doraiswamy NV: Retrograde ureteroplasty using balloon dilatation in children with pelviureteric obstruction. J Pediatr Surg 1994;29(7):937-940.

12. Sugita Y, Clarnette TD, Hutson JM: Retrograde balloon dilatation for primary pelvi ureteric junction stenosis in children. Br J Urol 1996;77(4):587-589.

13. Wilkinson AG, Azmy A: Balloon dilatation of the pelvi ureteric junction in children: Early experience and pitfalls. Pediatr Radiol 1996;26(12):882-886

14. MacKenzie RK, Youngson GG, Hussey JK, Mahomed AA: Is there a role for balloon dilatation of pelvi ureteric obstruction in children? J Pediat Surg 2002;37(6):893-896.

15. McClinton S, Steyn JH, Hussey JK: Retrograde balloon dilatation for pelviureteric junction obstruction. $\mathrm{Br} \mathrm{J}$ Urol 1993;71(2):152-155.

16. Kandir S, White RI Jr, Engel R: Balloon dilatation of ureteropelvic junction obstruction. Radiology 1982;143(1): 263264.

17. Bolton DM, Bogaert GA, Mevorach RA, Kogan BA, Stoller ML: Pediatric ureteropelvic junction obstruction treated with retrograde endopyelotomy. Urology 1994;44(4):609613.

18. Figenshau RS, Clayman RV: Endourologic options for management of ureteropelvic junction obstruction in the pediatric patient. Urol Clin Nort Am 1998;25(2):199-209.

19. Dewan PA, Ng KP, Ashwood PJ: The relationship of age to pathology in pelviureteric junction obstruction. J Paediatr Child Health 1998;34(4):384-386.

20. Nakada SY, Wolf JS, Brink JA, Quillen SP, Nadler RB, Gaines MV, et al: Retrospective analysis of the effect of crossing vessels on retrograde endopyelotomy outcomes using spiral CT angiography. J Urol 1998;159(1):62-65.

Dr. A. Parente Hernández

Hospital Materno-Infantil Gregorio Marañón

Servicio de Cirugía Pediátrica

Maiquez 9 , 4ำ F 11

28009 Madrid

E-mail: parente80@hotmail.com

(Trabajo recibido el 9 de junio de 2006) 